\title{
Time-Dependent Discrete Network Design Frameworks Considering Land Use
}

\author{
W. Y. Szeto* \\ Department of Civil Engineering, The University of Hong Kong, Pokfulam Road, Hong Kong, China
}

$\&$

Xiaoqing Jaber \& Margaret O'Mahony

Centre for Transport Research and Innovation for People (TRIP), Department of Civil, Structural and Environmental Engineering, Trinity College Dublin, Dublin 2, Ireland

\begin{abstract}
This article proposes optimization frameworks for discrete road network design considering the land-use transport interaction over time. Unlike existing models, the optimization frameworks can determine the optimal designs automatically without trial-and-error once the objective(s) is/are clearly defined. Moreover, these frameworks allow the evaluation of the impacts of the optimal designs on the related parties including landowners, toll road operators, transit operators, and road users, and help network planners and profit-makers with decision making by eliminating many alternative designs. A numerical study is set up to examine road network design's effects on these related parties under three road construction schemes: exact cost recovery, build-operate-transfer, and cross-subsidization. The results show that the changes in landowner profits are not the same after implementing any scheme. These unequal changes raise the issue of the landowner equity. This implies that the government has to consider trade-offs between parties' objectives carefully.
\end{abstract}

\section{INTRODUCTION}

Nowadays, many road improvement and construction projects are still ongoing, especially in some major

*To whom correspondence should be addressed. E-mail: ceszeto@ hku.hk. cities in Asia and Europe. These projects are expensive. With respect to constrained government expenditure, the government should carefully select cost-effective improvement and construction projects to be implemented. Traditionally, the analysis involved belongs to the discipline of road network design. In the past, much research (e.g., LeBlanc, 1975; Abdulaal and LeBlanc, 1979; Boyce and Janson, 1980; Boyce, 1984; Magnanti and Wong, 1984; Friesz, 1985; Marcotte, 1986; Chen and Alfa, 1991; Friesz et al., 1993; Davis, 1994; Meng et al., 2001; Chen and Yang, 2004; Chiou, 2005, 2007, 2009; Ban et al., 2006; Vitins and Axhausen, 2009) was done on this discipline via the static approach. Yang and Bell (1998) and Lo and Szeto (2009) provided comprehensive reviews on the static approach to this discipline.

Recently, researchers considered the time dimension in road network design. Three time scales are typically considered in the literature: seconds, days, and years. The smallest time scale (e.g., Heydecker, 2002) is used to capture the within-day dynamics such as queuing phenomena, the fluctuation of demand within a day, and the departure choice of travelers. The medium scale (e.g., Friesz and Shah, 2001) is used to capture the route adjustment behavior of travelers from day to day. The largest scale (e.g., Szeto and Lo, 2006, 2008; Lo and Szeto, 2009; Unnikrishnan et al., 2009) is used to capture the changing demand, gradual network upgrades, and cost and benefit over a long period of time, to maintain a similar social equity level over years, and to determine 
the optimal infrastructure improvement timetable, and its associated financial arrangement and tolling scheme.

Some studies (e.g., Smith and Liebman, 1978; Los, 1978, 1979; Peiser, 1984; Feng and Lin, 1999; Meng et al., 2000; Lin and Feng, 2003; Lee et al., 2006; Qiu and Chen, 2007) also incorporated land use into the analysis of the Network Design Problem (NDP). All previous efforts on the NDP, however, ignore the land usetransport interaction over time. In reality, the transport system interacts with the land-use system. When a new road is built or an existing road is widened, the travel costs between some zones decrease, and hence the accessibilities for those zones increase. Increases in the accessibilities lead to changes in population and employment distributions, and in turn a new travel demand pattern over time. The new travel demand pattern leads to a new traffic pattern and new congestion locations, which may require further improvements in the future. Ignoring this time-dependent land use-transport interaction may result in wrong allocations of budgets on road improvements or starting the improvements at wrong locations or at suboptimal times. In addition, the long-term impact of road improvement and construction policies to the land-use system and the benefit of landowners cannot be evaluated without considering the interaction.

This article develops a general time-dependent discrete network design framework encapsulating the Lowry-type land-use consideration so that the land usetransport interaction can be dealt with when determining the optimal design. Both link addition and link expansion are considered with capacity modeled as a discrete variable. Multimodal transport interaction is also captured in the framework. This article differs from Szeto and Lo (2006, 2008) and Lo and Szeto (2009) which do not consider land use and its interaction with transport, assume that capacity is a continuous variable, and consider only single transport mode. More importantly, using the proposed framework, we can study the effect of road improvement and construction policies (e.g., subsidization of road construction projects using public fund or transit revenue, cost recovery, and the build-operate-transfer (BOT)) on land use, the profits of landowners and thcir profit distribution as well as population and employment changes. The time scale we consider in the framework is in years as in Szeto and Lo (2006, 2008) and Lo and Szeto (2009), because the pace of the adjustment process inside the land-use system is slow compared with those that occurred inside the transport system like the day-to-day route adjustment process or the second-to-second traffic dynamics. Nonetheless, a second smaller time dimension can be easily added to the proposed models to cope with the dynamics inside the transport system without concep- tual difficulty and is left for future study. Because the largest time scale is used here, the inherent advantages of the model proposed by Szeto and Lo $(2006,2008)$ and Lo and Szeto (2009) can be found in this framework.

The framework is formulated as a single-level singleobjective optimization program, in which the objective function represents the concern of the decision maker. The decision maker can be the government or the private sector, depending on the source of funding for road improvement and construction projects. The objective thus depends on who is the decision maker or builder/operator. In the case where the funding is wholly from the government who is in charge of road network design, the decision maker is the government and their objective can be alleviating congestion or maximizing societal benefit. In the case where the improvement or construction projects involve the private sector (i.e., the builder and operator is the private sector) like BOT projects, the objective is usually profit-maximizing. The constraints include those describing modal split, trip assignment, land-use transport interaction over time, as well as design and financial constraints. To incorporate the considerations of various parties involved in road improvement and construction projects, a multi-objective optimization framework is then developed through the hybrid approach. This multi-objective framework can aid the government making decisions considering the objective of each related party and eliminating a large number of alternative designs without trial and error.

A numerical study using a small network is set up to illustrate the effect of the implementation of road construction projects on the related parties, especially on landowners, and show trade-offs between various objectives, although the models can be used to handle general networks. Three road construction schemes are considered: exact cost recovery, BOT, and to use the increase in transit profit to subsidize road construction projects. The scenario under each scheme is formulated individually using the proposed frameworks, and the corresponding optimal design is obtained by PREMIUM SOLVER PLATFORM to solve the models. The results show that the changes in landowner profits are not the same after implementing any one of three projects. This raises the issue of landowner equity in terms of changes in landowner profits. More importantly, the changes can be negative after the implementation. If we force the changes in landowner profits to be nonnegative, societal benefit can be reduced and the road network can be more congested compared with the situation without enforcing nonnegative changes in landowner profits.

The rest of the article is organized as follows: The next section describes the formulation of the 
single-objective framework and the framework extension. Following that is the numerical study. The last section gives concluding remarks.

\section{FORMULATIONS}

We consider a strongly connected multimodal transportation network with multiple origin-destination (OD) flows over the planning horizon $[0, T]$. The planning horizon is divided into $N$ equal design periods. The network is further divided into $M$ subnetworks, one for each mode, to account for the unique traveling speed of each mode. The mode here can be an individual mode or a combined mode. The proposed roads are also included in the bus, minibus, and car mode subnetworks. With this consideration, we can formulate the proposed framework as a single-level, single-objective constrained optimization program as follows:

$$
\max y(\mathbf{x})
$$

subject to time-dependent Lowry-type constraints,

$$
\text { time-dependent modal-split/ }
$$$$
\text { assignment constraints, }
$$

road network design constraints, and;

financial constraints

where $y(\mathbf{x})$ is the objective function and $\mathbf{x}$ is the vector of decision variables. In the following, we discuss the framework in detail.

\subsection{Time-dependent Lowry-type constraints}

Time-dependent Lowry-type constraints are developed based on Lowry's (1964) model, which classifies the land use into three categories: basic sector, household sector, and nonbasic sector. The basic sector includes industries, businesses, and administrative establishments whose goods and services are exported outside the urban area. It generates a centripetal flow of capital into the city generating growth and surpluses. It is generally assumed that this sector is less constrained by urban location problems because the local market is not the main concern. This consideration is an exogenous element of the Lowry model and must be given. The nonbasic sector includes those businesses, administrative, and other establishments that deal with providing goods and services for local residential population. Because this sector strictly serves the local/regional demand, the location choice is oriented to the household sector. Employment levels are also assumed to be linked with the local population. The household sector consists of residential population. The number of residents is related to the number of basic and nonbasic jobs available. Their residential locations are also closely linked to the place of work. Because the residential and nonbasic sector location choices depend on each other, the household and nonbasic sectors finally distribute themselves to achieve equilibrium.

The time-dependent Lowry-type constraints extend the Lowry-type equilibrium to a dynamic framework. In each design period, a Lowry-type equilibrium is assumed to be held. The equilibrium is depicted by a number of constraints. The first one describes how to allocate residents who work in employment zone $i$ to residential zone $j$ using the gravity-type model:

$$
\begin{gathered}
R_{i j, \tau}=E_{i, \tau} B_{i, \tau} W_{j, \tau}^{\alpha} \exp \left(-\beta^{r} c_{i j, \tau}\right), \forall i, j, \tau \\
\text { where } B_{i, \tau}=1 / \sum_{j} W_{j, \tau}^{\alpha} \exp \left(-\beta^{r} c_{i j, \tau}\right), \forall i, \tau
\end{gathered}
$$

$R_{i j, \tau}$ is the number of residents traveling between OD pair $i j$ in period $\tau$ or the number of work-to-home trips (or the number of total employment trips) between OD pair $i j$ in period $\tau$. This is the number of residents in zone $j$ that work in zone $i . E_{i, \tau}$ is the total employment in zone $i$ in period $\tau . W_{j, \tau}$ is the attractiveness of zone $j$ in period $\tau$, which can be represented by the availability of floor space for residential use. $\alpha$ is the economyof-scale parameter to regulate the attractiveness of each zone (Bureau of Transport Economics, 1998). $\beta^{r}$ is a parameter to regulate the effect of transport cost on distribution of residents. A high value of $\beta^{r}$ will result in the residents being allocated close to their places of work; if $\beta^{r}$ tends to infinity, all residents will live and work in the same zone. On the other hand, if $\beta^{r}$ tends to zero, the residents whose work in zone $i$ will locate to all residential zones equally. $c_{i j . r}$ is the composite travel cost between OD pair $i j$ in year $\tau$, representing the inter-zonal impedance and will be defined later. The balancing factor $B_{i, \tau}$ is derived by Wilson (1970), which is to ensure a correct allocation of residents to zone $j$ in period $\tau$ so that $\sum_{j} B_{i, \tau} W_{j, \tau}^{\alpha} \exp \left(-\beta^{r} c_{i j, \tau}\right)=1$ and the total number of work-to-home trips from employment zone $i$ in period $\tau$ must equal the number of jobs available in that zone in period $\tau, E_{i, \tau}$, or the number of people working in employment zone $i$ in period $\tau$ must equal the number of jobs available in that zone in the same period (i.e., $\sum_{j} R_{i j . \tau}=E_{i, \tau}$ ).

The total employment in zone $i$ in period $\tau, E_{i, \tau}$, in (2) is the sum of the basic employment $E_{i, \tau}^{B}$ and the service employment or nonbasic employment, $E_{i, \tau}^{S}$, in zone $i$ in period $\tau$ :

$$
E_{i, \tau}=E_{i, \tau}^{B}+E_{i, \tau}^{S}, \forall i, \tau
$$

The service employment in zone $i$ in period $\tau, E_{i, \tau}^{S}$, in (4) is equal to the number of service employment trips 
starting from zone $i$ in period $\tau$ or simply the number of service employees working there in that period:

$$
E_{i . \tau}^{S}=\sum_{j} E_{i j . \tau}^{S}, \forall i, j, \tau
$$

where $E_{i j . \tau}^{S}$ is the number of service employees who work in zone $i$ living in zone $j$ or the number of service employment trips between OD pair $i j$ in period $\tau$.

The number of service employment trips $E_{i j, \tau}^{S}$ is obtained by

$$
\begin{gathered}
E_{i j, \tau}^{S}=s R_{j, \tau} A_{j, \tau} W_{i, \tau}^{\alpha} \exp \left(-\beta^{s} c_{i j, \tau}\right), \forall i, j, \tau \\
\text { where } A_{j, \tau}=1 / \sum_{i} W_{i, \tau}^{\alpha} \exp \left(-\beta^{s} c_{i j, \tau}\right), \forall j, \tau
\end{gathered}
$$

$s$ is a service employment-to-population ratio. $W_{i, \tau}$ is the attractiveness in zone $i$ in period $\tau$, which can be the availability of floor space for commercial use. $\alpha$ is the economy-of-scale parameter to regulate the attractiveness of each zone. $\beta^{s}$ is a parameter to regulate the effect of transport cost on distribution of service employees, and its function is similar to $\beta^{r}$ in (2). A high value of $\beta^{s}$ will result in service employment being allocated close to the residential location, and a small value will result in service employment being allocated to all residential locations equally. The term $A_{j . r}$ is to ensure a correct allocation of service employment to zone $i$, which has a similar function to the term $B_{i, \tau}$ in (3). The term $s R_{j, \tau}$ in (6) is the total number of employees in zone $j$ in period $\tau$.

The total number of residents in zone $j$ in period $\tau$, $R_{j . \tau}$, in (6) is defined as

$$
R_{j, \tau}=\mu \sum_{i} R_{i j, \tau}, \forall j, \tau
$$

where $R_{i j . \tau}$ is the number of employees who work in $i$ living in $j$ in period $\tau$, and $\mu$ is a population-toemployment ratio. According to (8), in each period, the total number of employees in zone $j, \sum_{i} R_{i j, \tau}$ multiplied by the population-to-employment $\mu$ gives the total number of residents in that zone $R_{j, \tau}$.

\subsection{Time-dependent modal-split/assignment constraints}

The time-dependent modal-split assignment constraints represent the transport model in this framework and describe the route and mode choices over time. These constraints are made up of Wardrop's (1952) conditions, travel cost constraints, as well as the modal split, flow conservation, and nonnegativity conditions.

2.2.1 Wardrop's conditions. These conditions are supposed to be held in each design period for each mode.
They require that for each mode $k$ in each period $\tau$, the travel cost of each of the used routes between the same OD pair must be equal and minimal. This can be mathematically represented as

$$
f_{p, i j, \tau}^{k}\left\{\begin{array}{ll}
\geq 0 & \text { if } c_{p, i j, \tau}^{k}=\pi_{i j, \tau}^{k}, \\
=0 & \text { if } c_{p, i j, \tau}^{k}>\pi_{i j, \tau}^{k},
\end{array} \forall p, i, j, k, \tau\right.
$$

where $f_{p, i j, \tau}^{k}$ is the representative hourly flow of mode $k$ on route $p$ between OD pair $i j$ in period $\tau ; c_{p, i j, \tau}^{k}$ is the travel cost on route $p$ between OD pair $i j$ by mode $k$ in period $\tau$; and $\pi_{i j, \tau}^{k}$ is the lowest total travel cost between OD pair $i j$ by mode $k$ in period $\tau$ :

$$
\pi_{i j, \tau}^{k}=\min \left[c_{p, i j, \tau}^{k}, \forall p\right]
$$

According to (9) and (10), if the flow of mode $k$ on route $p$ between OD pair $i j$ in period $\tau$ is larger than zero, its travel cost is the lowest travel cost. If the travel cost of mode $k$ on route $p$ in period $\tau$ between OD pair $i j$ is larger than the lowest travel cost, the flow of mode $k$ is zero.

Condition (9) can be restated as

$$
\begin{gathered}
f_{p, i j, \tau}^{k}\left[c_{p, i j, \tau}^{k}-\pi_{i j, \tau}^{k}\right]=0, \forall p, i, j, k, \tau \\
c_{p, i j, \tau}^{k}-\pi_{i j, \tau}^{k} \geq 0, \forall p, i, j, k, \tau
\end{gathered}
$$

2.2.2 Travel cost constraints. Travel costs depend on flows, network characteristics such as free flow travel times and capacities of links, and out-of-pocket costs such as tolls and fares. Route costs depend on route flows, in which the latter depend on link flows through:

$$
v_{a, \tau}^{k}=\sum_{i j} \sum_{p} f_{p, i j, \tau}^{k} \delta_{a}^{p, k}, \forall a, k, \tau
$$

where $v_{a, \tau}^{k}$ is the hourly flow of mode $k$ on link $a$ in pe$\operatorname{riod} \tau$, and $\delta_{a}^{p, k}$ is a link-path incidence indicator for mode $k$, equals one if link $a$ is on route $p$, zero otherwise. Equation (13) states that for each mode $k$, the link flow in each period is obtained by summing the corresponding route flows on that link in that period.

The link time $t_{a, \tau}^{k}$ (such as travel time, waiting time, or walking time) relates link flows through the link performance function:

$$
t_{a, \tau}^{k}=t_{a, \tau}^{k}\left(\mathbf{v}_{\tau}\right)
$$

where $\mathbf{v}_{\tau}=\left[v_{a, \tau}^{k}\right]$ is the link flow vector in period $\tau$. This link performance function is nonseparable as the link time on link $a$ depending on the flows on other links. Because the focus of this article is on road network 
design, we give an example of the link performance function commonly used in road traffic assignment:

$$
\begin{gathered}
t_{a, \tau}^{m}=t_{a}^{0, m}\left[1+\left(\frac{\sum_{a} \sum_{k} \alpha_{a, k} v_{a, \tau}^{k}}{\bar{c}_{a, \tau}^{m}}\right)^{\gamma_{a, m}}\right], \forall a, m, \tau \\
\bar{c}_{a, \tau}^{m}=c_{a}^{m, 0}+\sum_{b} \sum_{\omega=1}^{\tau} \delta_{a, b}^{m} y_{b, \omega}, \forall a, m, \tau \\
y_{b, \tau}=0, S, 2 S, 3 S, \ldots, \forall b, \tau
\end{gathered}
$$

where the superscript $m$ stands for the mode that travels in the road network only (which is different from the superscript $k$ that is used for representing any mode considered in this article). The symbols $t_{a}^{0, m}$ and $c_{a}^{0, m}$ denote the free flow travel time and initial capacity of link $\boldsymbol{a}$ used by mode $m$ that travels on roads. For a proposed new link, $c_{a}^{0, m}=0$. The symbols $\alpha_{a, k}$ and $\gamma_{a, k}$ represent the parameters of the performance function of link $a$ for mode $m$. The symbol $\bar{c}_{a, \tau}^{m}$ is the capacity of link $a$ used by mode $m$ in period $\tau$ whereas $S$ is the capacity of a standard lane. The variable $y_{b, \tau}$ represents the capacity improvement on link $b$ in period $\tau$, meaning that the capacity of link $b$ is increased by $y_{b . \tau}$ units at the beginning of period $\tau$. The symbol $\delta_{a, b}^{m}$ is the simultaneous improvement/construction indicator, which equals one if the improvement/construction on link $b$ (in which $b$ stands for a particular road) will also result in improvement/construction on link $a$ used by mode $m$ or $a=b$, and zero otherwise. This indicator is used in (16) because a street or road may be used by two different modes, say buses and cars, with different traveling speeds and we model the street or road by two different links with the same capacity.

Equation (15) is an asymmetric link performance function, which describes the monotonic relationship between the link travel time $t_{a, \tau}^{m}$ and the link flows $v_{a, \tau}^{k}$. When $\alpha_{a, m}=0.15, \alpha_{b, k}=0, a \neq b, m \neq k$, and $\gamma_{a, m}=4$, Equation (15) is reduced to the typical Bureau of Public Roads (BPR) function. Equation (16) expresses the capacity of link $a$ used by mode $m$ in period $\tau$ as the sum of its initial capacity $c_{a}^{m, 0}$ and total improvements up to period $\tau, \sum_{b} \sum_{\omega=1}^{\tau} \delta_{a, b}^{m} y_{b, \omega}\left(\right.$ or $\sum_{\omega=1}^{\tau} y_{a, \omega}$ when $\left.a=b\right)$. Equation (17) constrains capacity enhancements to be the multiple of the capacity of a standard lane.

The route cost $c_{p . i j, \tau}^{k}$ is the sum of the link-wise additive costs $g_{p, i j, \tau}^{k}$ and the route specific $\operatorname{costs} \theta_{p, i j, \tau}^{k}$ :

$$
c_{p . i j, \tau}^{k}=g_{p . i j, \tau}^{k}+\theta_{p . i j, \tau}^{k}, \forall p, i, j, k, \tau
$$

The link-wise additive costs $g_{p, i j, \tau}^{k}$ are defined by summing up link attributes, which include the link tolls $\rho_{a . \tau}^{k}$ and congestion-dependent attributes; for instance, travel time (and other costs such as fuel consumption) for road networks, or walking, on-board and boarding/alighting time for transit networks. The link-wise additive cost $g_{p, i j, \tau}^{k}$ can be written as

$$
g_{p, i j, \tau}^{k}=\sum_{a}\left(\psi t_{a, \tau}^{k}+\rho_{a, \tau}^{k}\right) \cdot \delta_{a}^{p, k}, \forall p, i, j, k, \tau
$$

where $\psi$ is the cost of unit (travel) time, and therefore $\psi t_{a, \tau}^{k}$ is the (travel) time cost on link $a$ by mode $k$ in period $\tau$. The variable $\rho_{a, \tau}^{k}$ represents the toll for mode $k$ using link $a$ in period $\tau$.

The route specific costs $\theta_{p, i j, \tau}^{k}$ are nonlinear and/or nonadditive over links; for instance, some types of tolls in road networks (e.g., nonlinearly proportional to distance), or waiting time and some fare structures for transit networks (e.g., zone-wise prices).

The composite travel cost between OD pair $i j$ in period $\tau, c_{i j . \tau}$, is defined as

$$
c_{i j, \tau}=-\ln \left[\sum_{k}\left(\exp \left(-\bar{\beta}\left(\pi_{i j, \tau}^{k}+\theta^{k}\right)\right)\right] / \bar{\beta}, \forall i, j, \tau\right.
$$

where $\bar{\beta}$ is the parameter in the logit model to regulate the effect of the mode travel cost $\pi_{i j, \tau}^{k}+\theta^{k}$. The parameter $\theta^{k}$ denotes the mode-specific cost. The composite cost is obtained by aggregating the mode travel $\operatorname{cost} \pi_{i j, \tau}^{k}+\theta^{k}$ over all modes $k$. The derivation of this composite cost can be found in Williams (1977) and Ben Akiva and Lerman (1987).

2.2.3 The modal split, flow conservation, and nonnegativity conditions. Modal split can be obtained by the logit model:

$$
q_{i j, \tau}^{k}=R_{i j, \tau}\left(\frac{\exp \left(-\bar{\beta}\left(\pi_{i j, \tau}^{k}+\theta_{i j}^{k}\right)\right)}{\sum_{m} \exp \left(-\bar{\beta}\left(\pi_{i j, \tau}^{m}+\theta_{i j}^{m}\right)\right)}\right), \forall i, j, k, \tau
$$

where $q_{i j, \tau}^{k}$ is the demand for mode $k$ between OD pair $i j$ in period $\tau$, and $R_{i j . \tau}$ is the number of residents who work in zone $i$ living in $j$ defined in (2).

The demand for mode $k$ between OD pair $i j$ in period $\tau, q_{i j, \tau}^{k}$, in (21) is equal to the sum of the route flows of that mode between the OD pair in the same period so that route flows are conserved in each mode between each OD pair in each period:

$$
q_{i j, \tau}^{k}=\sum_{p} f_{p, i j, \tau}^{k}, \forall i, j, k, \tau
$$

Moreover, route flow in (22) must be nonnegative:

$$
f_{p, i j . \tau}^{k} \geq 0, \forall p, i, j, k, \tau
$$

\subsection{Road network design constraints}

They include link addition constraints, capacity constraints, and toll constraints. 
2.3.1 Link addition constraints. These constraints are added to ensure that flow on a proposed link can be nonnegative only if the link is built, and is zero otherwise:

$$
v_{a, \tau}^{m} \leq M \sum_{b} \sum_{\omega=1}^{\tau} \delta_{a, b}^{m} y_{b, \omega}, \forall a, m, \tau
$$

where $M$ is a very large constant.

2.3.2 Capacity constraints. These constraints are included to address the facts that a link (in road networks) cannot be built or expanded beyond an upper limit due to space limitation:

$$
\bar{c}_{a, \tau}^{m} \leq u_{a}^{m}, \forall a, m, \tau
$$

where $u_{a}^{m}$ is the maximum allowable capacity of link $a$ for mode $m$ that uses the highways or roads. Equation (25) is the maximum allowable capacity constraint, which is to limit the total capacity of each link after road expansion or highway construction in period $\tau, \bar{c}_{a . \tau}^{m}$, to be less than its maximum allowable capacity.

2.3.3 Toll constraints. These constraints cater for scenarios, such as for political reasons, the toll $\rho_{l, \tau}^{m}$ cannot be collected on certain links, or toll charges cannot be set too high. Mathematically, they can be stated as

$$
\begin{gathered}
\rho_{l . \tau}^{m}=0, \forall l, m, \tau \\
\rho_{a . \tau}^{m} \geq 0, \forall a, m . \tau \\
\rho_{a . \tau}^{m} \leq \rho_{\max }^{m}, \forall a, m, \tau
\end{gathered}
$$

where $\rho_{\max }^{m}$ is the maximum allowable toll for mode $m$ that travels on roads. The subscript $l$ represents links without tolls.

\subsection{Financial constraints}

They depict the relationship between the improvement and construction costs, toll revenues, and subsidy. These constraints include cost and revenue functions and the cost recovery constraint.

2.4.1 Cost and revenue functions. The toll revenue $T_{\tau}$, and the improvement, construction, and maintenance cost $K_{\tau}$ in period $\tau$ can be in terms of the equilibrium link flow $v_{a, \tau}^{m}$, the toll $\rho_{a, \tau}^{m}$, and the improvement $y_{b, \tau}$ as follows:

$$
\begin{aligned}
T_{\tau} & =\sum_{m} \sum_{a} n v_{a, \tau}^{m} \rho_{a, \tau}^{m}, \forall \tau \\
K_{\tau} & =\sum_{b}\left(h_{b . \tau}+w_{b . \tau}\right), \forall \tau
\end{aligned}
$$

$$
\begin{gathered}
h_{b, \tau}=(1+\tilde{j})^{\tau-1} \bar{b}_{b, 0} l_{b}\left(y_{b, \tau}\right)^{\bar{b}_{b .1}}, \forall b, \tau \\
w_{b, \tau}=(1+\tilde{j})^{\tau-1}\left[\beta_{b, 0}+\beta_{b, 1}\left(\sum_{m}\left(n v_{b, \tau}^{m}\right)\right)^{\beta_{b, 2}}\right], \forall b, \tau
\end{gathered}
$$

where $h_{b, \tau}$ and $w_{b, \tau}$ are the improvement, construction, and maintenance cost functions of link $b$ in period $\tau$, respectively. The symbols $\bar{b}_{b, 0}, \bar{b}_{b, 1}, \beta_{b, 0}, \beta_{b, 1}$, and $\beta_{b, 2}$ are the parameters of these cost functions. The parameter $n$ converts link flows from an hourly basis to a period basis and $\tilde{j}$ is the inflation rate. The symbol $l_{b}$ represents the length of link $b$. Equation (29) calculates the toll revenue in period $\tau$, which is the sum of the product of the link flow and toll in that period. Equation (30) computes the improvement, construction, and maintenance cost in period $\tau$ by adding the improvement, construction, and maintenance costs of all links. Equation (31) is the time-dependent improvement and construction cost function. The term $(1+\tilde{j})^{\tau-1}$ represents the inflation factor: for the same capacity enhancement, the improvement and construction cost increases by $100 \tilde{j} \%$ each period. The term $\bar{b}_{b, 0} l_{b} y_{b, 1}^{b_{b, 1}}$ models the improvement and construction cost of link $a$ in period 1 (i.e., the base period). Equation (31) depicts the general relationship that the improvement and construction cost of a link is proportional to the extent of the widening (and hence capacity gain) and its length. This function is adopted for illustration and simplicity; other functional forms can be adopted in this framework without difficulty. Equation (32) is the time-dependent maintenance cost function, which is set to be: $\beta_{b, 0}+\beta_{b, 1}\left(\sum_{m}\left(n v_{b, 1}^{m}\right)\right)^{\beta_{b, 2}}$ in the base period. This function consists of the fixed cost $\beta_{b, 0}$ and the variable cost $\beta_{b, 1}\left(\sum_{m}\left(n v_{b, \tau}^{m}\right)\right)^{\beta_{b, 2}}$, in which $\sum_{m}\left(n v_{b, \tau}^{m}\right)$ is the link flow on link $b$ in period $\tau$. Again, the maintenance cost depends on the infiation factor $(1+\tilde{j})^{\tau-1}$.

2.4.2 Cost recovery constraints. Cost recovery can be classified into three types: partial, exact, and profitable (Lo and Szeto, 2009). Partial (exact) cost recovery occurs when the cost in a design period is partially (exactly) recovered by the revenue, adjusted to present value terms. Profitable cost recovery occurs when, in present value terms, the revenue more than covers the cost, with a surplus or profit at the end of the planning horizon. These three cost recovery schemes can be mathematically formulated using one equation:

$\sum_{\tau} \frac{T_{\tau}}{(1+\tilde{i})^{\tau-1}}+\sum_{\tau} \frac{S_{\tau}}{(1+\tilde{i})^{\tau-1}}-\sum_{\tau} \frac{K_{\tau}}{(1+\tilde{i})^{\tau-1}}=T O P$

where $S_{\tau}$ is the subsidy or contribution to network improvements in period $\tau$ and $T O P$ is the profit or surplus 
of the toll road operator. The parameter $\tilde{i}$ denotes the discount rate. The term $\frac{1}{(1+i)^{r-1}}$ is the discount factor for period $\tau$.

The first term on the left-hand side (LHS) of (33) is the total discounted toll revenue for the entire planning horizon. Similarly, the second (third) term is the total discounted government subsidy (the total discounted improvement, construction, and maintenance cost). The cost recovery equation (33) requires that, in present value terms, the total toll revenue plus the total subsidy minus the total improvement, construction, and maintenance cost equals the surplus or profit. Depending on the values of $S_{\tau}$ and TOP, Equation (33) reduces to (1) the partial cost recovery equation if $S_{\tau}$ is positive and $T O P$ is zero, (2) the exact cost recovery equation if all $S_{\tau}$ and TOP are zcro, or (3) the profitablc cost recovery equation if all $S_{\tau}$ are zero and TOP is positive.

In the case where the subsidy $S_{\tau}$ is obtained from the increase in transit profit (which is numerically the same as the increase in transit revenue when the operation and maintenance cost is fixed) $\Delta U_{\tau}^{k^{\prime}}$, the subsidy can be calculated by

$$
\begin{gathered}
S_{\tau}=\Delta U_{\tau}^{k^{\prime}}=U_{\tau}^{k^{\prime} \text {.after }}-U_{\tau}^{k^{\prime} \text {.before }}, \text { and } \\
U_{\tau}^{k^{\prime}}=\sum_{i j} \sum_{p} p_{p, i j, \tau}^{k^{\prime}} n f_{p, i j, \tau}^{k^{\prime}}, \forall \tau, k^{\prime}
\end{gathered}
$$

where the superscripts "before" and "after" denote before and after improvement and construction project implementations, respectively. The symbols $p_{p . i j, \tau}^{k^{\prime}}$ and $U_{\tau}^{k^{\prime}}$ denote respectively, the fare and revenue of transit mode $k^{\prime}$ on route $p$ between OD pair $i j$ in period $\tau$ whose profit is used to subsidize road improvements and constructions. Equation (35) states that the subsidy due to the revenue of transit mode $k^{\prime}$ is the sum of the product of the fare, $p_{p, i j . \tau}^{k^{\prime}}$ and the corresponding passenger flow $n f_{p, i j, \tau}^{k^{\prime}}$ in the period considered.

\subsection{Objective function}

The objective depends on who is the decision maker. In the case where the improvement and construction projects involve the private sector (i.e., the builder and operator are the private sector) like BOT projects, the objective is usually profit-maximizing, and the objective function is $T O P$ defined by (33).

In the case where the funding is wholly from the government who is in charge of a road network design, the decision maker is the government who usually considers a number of objectives from the viewpoint of society. The main one is societal benefit, or equivalently the change in societal benefit after implementing a transport policy like implementing a road construction project (because the societal benefit before the implementation is a constant that does not affect finding the optimal design during optimization). This can be measured by the change in social surplus (SS).

2.5.1 The change in social surplus. The change in social surplus, $\triangle S S$, is the difference between the SS after and before the implementation, and is equal to the sum of the change in consumer surplus (CS), $\triangle C S$, the change in landowner profit, $\triangle L O P$, the change in toll revenue, $\Delta T$, the change in transit revenue, $\Delta U$, minus the change in net tax revenue, $\Delta R$, the change in improvement, construction, and maintenance cost for the toll road, $\Delta K$, and the change in operation and maintenance cost of transit modes, $\Delta Y$ :

$$
\Delta S S=\Delta C S+\triangle L O P+\Delta T+\Delta U-\Delta R-\Delta K-\Delta Y
$$

The change in consumer surplus, $\triangle C S$, in Equation (36) measures the difference between what consumers would be willing to pay for travel and what they actually pay. It internalizes the effect of network congestion and the public's propensity to travel. For the same network and demand characteristics, a higher CS (positive change in CS) implies a better performing system. Here, an approximation to this change, in present value terms, is employed (Williams, 1976) and can be expressed as follows:

$$
\begin{aligned}
\Delta C S & =\sum_{\tau} \sum_{i j} \sum_{k} \frac{\Delta C S_{i j, \tau}^{k}}{(1+\tilde{i})^{\tau-1}} \\
\Delta C S_{i j, \tau}^{k}= & (1 / 2)\left(q_{i j, \tau}^{k, \text { before }}+q_{i j, \tau}^{k, \text { after }}\right) \\
& \times\left(\pi_{i j, \tau}^{k, \text { before }}-\pi_{i j, \tau}^{k, \text { after }}\right), \forall i, j, k, \tau
\end{aligned}
$$

According to (37), the change in CS is the sum of the change in CS for all modes and for all OD pairs over time, discounted to present value terms. Equation (38) is the rule-of-half definition for CS.

The change in landowner profit $\triangle L O P$ is the sum of the change of each individual discounted landowner profit $\triangle L O P_{j, \tau}$ over time:

$$
\triangle L O P=\sum_{\tau} \sum_{j} \frac{\Delta L O P_{j, \tau}}{(1+\tilde{i})^{\tau-1}}
$$

The difference of a landowner's profit before and after the implementation of a road improvement or construction project can be written as follows:

$$
\triangle L O P_{j, \tau}=L O P_{j, \tau}^{\text {atter }}-L O P_{j, \tau}^{\text {before }}, \forall j, \tau
$$

where $L O P_{j, \tau}^{\text {before }}$ and $L O P_{j, \tau}^{\mathrm{after}}$ represent the profits of landowner $j$ before and after the implementation of the road improvement/construction project in period $\tau$, The 
Landowner Profit (LOP) in residential zone $j$ in period $\tau$ can be expressed as follows:

$$
L O P_{j . \tau}=R_{j, \tau} r_{j, \tau}-M_{j . \tau}^{H}, \forall j, \tau
$$

where $R_{j . \tau}$ is the total number of residents in zone $j$ in period $\tau$ as in Equation (8) and $r_{j . \tau}$ is house rent per resident in residential zone $j$ in period $\tau$. The maintenance $\operatorname{cost}, M_{j . \tau}^{H}$, on houses can be formulated as a linear function as follows:

$$
M_{j, \tau}^{H}=\tilde{M}_{j . \tau}+m^{h} R_{j . \tau}, \forall j, \tau
$$

where $\tilde{M}_{j, r}$ is the fixed maintenance cost on houses in residential zone $j$ in period $\tau$ and $m^{h}$ is a parameter. The rent $r_{j, \tau}$ in (41) is assumed to increase over time due to inflation:

$$
r_{j . \tau+1}=r_{j . \tau}(1+\tilde{j})
$$

where $\tilde{j}$ is the inflation rate. This rent is fixed within a period as the housing supply is assumed to be perfectly elastic. This assumption can be relaxed in future studies.

The change in toll revenue $\Delta T$ can be similarly calculated by

$$
\Delta T=\sum_{\tau} \frac{\left(T_{\tau}^{\text {after }}-T_{\tau}^{\text {before }}\right)}{(1+\tilde{i})^{\tau-1}}
$$

The term in the numerator is the difference of toll revenue after and before the implementation of road improvement and construction projects. This term is discounted by $\frac{1}{(1+i)^{r-1}}$ to form the discounted change in toll revenue in period $\tau$. The sum of the discounted change in toll revenue in all periods is the change in toll revenue according to (44).

The changes in transit revenue, tax revenue, and the improvement, construction, and maintenance cost for toll roads can be defined in a way similar to the change in toll revenue, where the subsidy used to calculate the change in tax revenue and the total improvement, construction, and maintenance cost are zero before the implementation.

The change in operation and maintenance cost of transit $\Delta Y$ is zero, assuming that this cost is fixed and independent of the number of passengers.

\subsection{Considerations in road improvement and construction projects}

Developing a specific model requires taking into account the parties involved in the implementation of road network projects. In general, the implementations of road network projects involve many parties, including road users, private landowners, private transit operators, private toll road operators, and the government. Each of these parties has distinctive objectives as discussed below.
2.6.1 Road users: The shortest travel time and the lowest travel cost. Travelers are concerned with their actual travel times and travel costs. The actual travel time is the shortest travel time between an OD pair:

$$
\begin{gathered}
\tilde{g}_{i j, \tau}^{k}=\min \left[\hat{g}_{p, i j, \tau}^{k}, \forall p\right], \forall i, j, k, \tau \\
\text { where } \hat{g}_{p, i j, \tau}^{k}=\sum_{a} t_{a, \tau}^{k} \delta_{a}^{p, k}, \forall p, i, j, k, \tau
\end{gathered}
$$

The symbol $\tilde{g}_{i j, \tau}^{k}$ denotes the shortest travel time for mode $k$ between OD pair $i j$ in period $\tau$ and $\hat{g}_{p, i j, \tau}^{k}$ is the travel time of mode $k$ on path $p$ between OD pair $i j$ in period $\tau$. The travel cost is the lowest travel cost, $\pi_{i j, \tau}^{k}$.

2.6.2 Private landowners: Discounted profit or the change in landowner's (discounted) profit. In the case of private landowners, they are concerned with their own total discounted profit, which is the sum of the discounted landowner profit in each year. This can be formulated as

$$
L O P_{j}=\sum_{\tau} \frac{L O P_{j, \tau}}{(1+\tilde{i})^{\tau-1}}, \forall j
$$

where $L O P_{j}$ is the discounted profit of landowner $j$, and $L O P_{j, \tau}$ follows the definition in (41)-(42).

After the project implementation, there must be a change in landowner profit due to redistribution of residents. This change in landowner's profit can be used as an alternative to formulate the objective of the landowner, because the profit before the implementation is fixed. This change can be written as

$$
\Delta L O P_{j}=\sum_{\tau} \frac{\Delta L O P_{j, \tau}}{(1+\tilde{i})^{\tau-1}}, \forall j
$$

where $\triangle L O P_{j, \tau}$ is defined in (40)-(42). A positive value of $\triangle L O P_{j}$ means that the project implementation is beneficial to the landowner, and vice versa.

2.6.3 Private transit operators: Profit. Like private landowners, the objectives of transit operators are profit driven. The profit of the private transit operator can be written as

$$
U_{k^{\prime}}=\sum_{\tau} \frac{U_{\tau}^{k^{\prime}}-Y_{\tau}^{k^{\prime}}}{(1+\tilde{i})^{\tau-1}}, \forall k^{\prime}
$$

where $U_{\tau}^{k^{\prime}}$ is the revenue of transit operator $k^{\prime}$ in period $\tau$ defined in (35), and $Y_{\tau}^{k^{\prime}}$ is the operation and maintenance cost of transit operator $k^{\prime}$ in period $\tau$.

2.6.4 Private toll road operators: Profit. The objective of each private toll road operator is to maximize total discounted profit $T O P_{b}$, which is the difference 
between the total discounted revenue $T_{b}$ and the total discounted cost $K_{b}$ :

$$
\begin{gathered}
\operatorname{TOP}_{b}=T_{b}-K_{b}, \forall b \\
\text { where } T_{b}=\sum_{\tau} \sum_{m} \frac{n v_{a, \tau}^{m} \rho_{a, \tau}^{m}}{(1+\tilde{i})^{\tau-1}}, \forall b \\
K_{b}=\sum_{\tau} \frac{h_{b, \tau}}{(1+\tilde{i})^{\tau-1}}, \forall b
\end{gathered}
$$

The subscript $b$ represents the toll road operator. The term $n v_{a, \tau}^{m} \rho_{a, \tau}^{m}$ represents the toll revenue in period $\tau$ from mode $m$ on road networks, and $h_{b, t}$ is the improvement cost following earlier definition.

2.6.5 Government: Average network travel time and equity between landowners. The government has a lot of concerns, including the whole societal benefit, the congestion problem, the environmental issue, the equity issues between travelers, between private toll road operators, and between landowners, and so on. Here we only discuss and formulate two measures: the average network travel time and the equity constraints between landowners.

2.6.6 Average network travel time (ANTT). The average network travel time is defincd as follows:

$$
A N T T=\frac{\sum_{\tau} \sum_{k} \sum_{a} v_{a, \tau}^{m} t_{a, \tau}^{m}}{\sum_{\tau} \sum_{m} \sum_{a} v_{a, \tau}^{m}}
$$

The numerator is the total travel time of all modes using road networks over the planning horizon, whereas the denominator is the total traffic ovcr the planning horizon. This measure only considers the average speed of all modes on a road network and hence the congestion level of the network, which is different from consumer surplus that considers both the effect of network congestion and the public's propensity to travel.

2.6.7 Equity between landowners. In general, the implementation of road improvement projects may result in different changes in landowner profits. Some changes can be greater than the other, and some changes can be even negatives. This raises the issue of equity between landowners. Here we consider that simplest case of equity: all changes must be nonnegative. That is,

$$
\triangle L O P_{j} \geq 0, \forall j
$$

If all changes are nonnegative, we say that inequity does not exist. Although we notice that this is not the only way to define landowner equity, it is sufficient to illustrate that inequity exists between landowners due to network improvement and expansion, as will be seen in Section 3.

\subsection{Three models derived from the proposed single-objective framework}

Based on the considerations above as well as different combinations of objective functions and constraints discussed above, we can develop many specific singleobjective optimization models. In this section, three specific models are provided, which will be used in the numerical study. They are the profit maximization model, the social surplus maximization model under exact cost recovery, and the social surplus maximization model under cross-subsidization:

2.7.1 Profit maximization model (PM model). The profit maximization model can be obtained by setting $y(\mathbf{x})$ in (1) to be TOP defined by (33):

$$
\max _{\mathbf{E}, \mathbf{R}, \mathbf{f}, \mathbf{y}, \boldsymbol{\rho}} T O P
$$

subject to time-dependent Lowry-type constraints

(2)-(8),

time-dependent modal-split/assignment constraints (10)-(23),

road network design constraints (25)-(28), and; financial constraints (29)-(31),

where $\mathbf{E}, \mathbf{R}, \mathbf{f}, \mathbf{y}, \rho$ represent, respectively, the vectors of the number of service employment trips, the number of work-to-home trips, path flows, capacity improvement, and tolls. Note that the cost recovery condition (33) is included in the objective function rather than in financial constraints. This model is suitable to aid decision making in the BOT projects.

2.7.2 Cost recovery model ( $C R$ model). This can be formulated as follows:

$$
\max _{\mathbf{E}, \mathbf{R}, \mathbf{f}, \mathbf{Y}, \rho} \Delta S S
$$

subject to the same constraints as in the PM model, and,

the cost recovery condition (33) with

$$
T O P=0 \text { and } S_{\tau}=0, \forall \tau
$$

where $\Delta S S$ is defined by (36)-(44). This model formulates the problem from the government's perspective, assuming the toll revenue generated to be able to recover the improvement and maintenance cost. In the case when the improvement and maintenance cost is very expensive and the toll revenue generated is not able to recover the cost, the model gives no improvement, zero toll charges, and no change in SS. 
2.7.3 Cross-subsidization model (CS model). This can be formulated as follows:

$$
\max _{\text {E.R. . .y.p }} \Delta S S
$$

subject to the same constraints as in the CR model,

$$
\text { except } T O P \geq 0 \text { and } S_{\tau}>0, \forall \tau \text {, }
$$

the cross-subsidization conditions (34)-(35)

where $\mathbf{p}$ is the vector of transit fares. This model also formulates the problem from the perspective of the government, assuming that there is a transit profit and the increase in profit is enough to build the toll road. In reality, the change in transit profit can be negative but the transit operator can still have a profit. In this case, the profit can still be used to subsidize the toll road construction and its maintenance but the crosssubsidization condition requires modifications.

\subsection{Model extension: Multi-objective optimization}

2.8.1 Multi-optimization framework. The above singleobjective optimization model may not be able to give a design that makes every party happy, as will be shown in the numerical study. If this happens, we find a compromised design using the following multi-objective optimization framework extended from the proposed framework discussed before:

$$
\max \sum_{i} w_{i} y_{i}(\mathbf{x})
$$

subject to the same constraints as the single-objective framework

$$
\begin{gathered}
\sum_{i} w_{i}=1 \\
w_{i} \geq 0 \\
\tilde{y}_{j}(\mathbf{x}) \geq \varepsilon
\end{gathered}
$$

where $y_{i}(\mathbf{x})$ is the $i$ th (normalized) objective function. $\mathbf{x}$ is the vector of decision variables. The parameter $w_{i}$ denotes the (normalized) weight for the $i$ th objective function, which is an input. The relative magnitudes of all weights represent the relative importance of the corresponding objectives. The parameter $\varepsilon$ represents the aspiration level or the satisfactory objective value, and $\tilde{y}_{j}(\mathbf{x})$ is the $j$ th objective function that does not appear in the weighted objective function in (55).

In the above framework, the objective function (55) is formed by summing all the weighted objective functions. Condition (56) is the weight constraint, which requires the sum of all weights to be one to normalize all the weights. Condition (57) is the nonnegativity condition of the weights. Condition (58) is the performance constraint (or $\varepsilon$-constraint), which considers the objective that does not include in (55). The objective function is set to be greater than the desirable or satisfactory objective value to ensure that at optimality, the $j$ th objective value is at least equal to the satisfactory value.

\subsubsection{Cost recovery model under equity consideration} (CR-equity model). This multi-objective optimization model will be used in the numerical study and is formulated as follows:

$$
\max _{\mathbf{E}, \mathbf{R}, \mathbf{f}, \mathbf{y}, \rho} \Delta S S
$$

subject to the same constraints as in the $\mathrm{CR}$ model, and; the landowner equity constraint (54),

where $\Delta S S$ is defined by (36)-(44). The key difference between this model and the CR model is that this model has the landowner equity constraints, avoiding reduction in landowner profit due to the implementation of network improvement projects. However, incorporating these performance constraints in the singleobjective optimization can reduce the optimal objective value, which will be seen in the numerical study.

\section{NUMERICAL STUDIES}

This study is set up to compare the three schemes of road network design, namely, BOT, cost recovery, and cross-subsidization; illustrate the impacts of the implementation of road construction projects on the related parties, especially on landowners; and show the tradeoffs between various objectives of the related parties. The BOT scheme allows a private company to build a toll road and collect tolls to recover the construction and maintenance cost within a franchised period; and after the franchised period is over, all these toll roads are transferred back to the government. This scheme is very common now in Asia and Europe. The exact cost recovery scheme uses toll revenue to $e x$ actly recover the construction and maintenance cost. The tolling and construction strategy is to maximize the change in SS, rather than to maximize the profit as in the BOT scheme. Because the objective of this scheme is to maximize the change in SS, the private sector is not willing to be involved. The builder and operator is thus the government. This scheme can be found in India. The cross-subsidization scheme is similar to the exact cost recovery scheme except that the increase in transit profit is used to subsidize the construction and maintenance cost of the toll road. This scheme is not common and only applicable to a place like Ireland, where the 


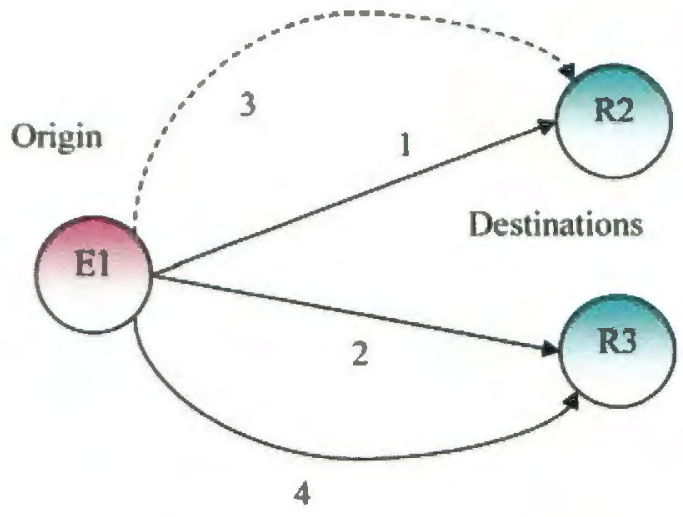

Fig. 1. The scenario network.

transit system is government-owned and can generate a huge profit due to high service coverage, high population, and low fare.

\subsection{Scenario setting}

Although the frameworks are applicable for large networks, a simple network is adopted as shown in Figure 1 for the ease of illustration. There are four links in this network: links 1-4. Links 1, 2, and 4 are links that have travel times given by the BPR functions and link 2 is a proposed new link. Link 3 is a separate transit link, as represented by a dashed line in the figure. There are 3 zones too: $E 1, R 2$, and R3, in which " $E$ " stands for an employment zone whereas " $R$ " stands for a residential zone. The attractiveness of each zone is assumed to follow the following function:

$$
W_{i, \tau+1}^{\alpha}=W_{i, \tau}^{\alpha}\left(1+\tilde{h}_{w, i}\right)
$$

where $h_{w, i}$ is the growth rate of attractiveness of zone $i$ over time. The basic employment in the employment zone is supposed to grow linearly over time:

$$
E_{i, \tau+1}^{B}=E_{i, \tau}^{B}\left(1+h_{E, i}\right)
$$

where $\tilde{h}_{E, i}$ is the growth rate of basic employment. The three zones form two OD pairs: E1-R2 and E1-R3. Both OD pairs are connected by highways but only OD pair E1-R2 has a separate transit connection. In other words, there are two modes for OD pair E1-R2 but there is only one mode for OD pair E1-R3.

The parameters in this study include:

1. Land-use parameters: $\tilde{h}_{w, 1}=\tilde{h}_{w, 2}=\tilde{h}_{w, 3}=0.05$; $h_{E, 1}=0.04 ; r_{2,0}=r_{3,0}=12 \times € 1,000 \times 10$ years $=$ $€ 120,000 ; E_{1.1}^{B}=5,000$ jobs; $W_{1,1}=3,000$ jobs; $W_{2,1}=W_{3,1}=3,000$ houses; $\alpha=1 ; \beta^{r}=0.04 €^{-1}$; $\beta^{s}=0.03 €^{-1} ; \mu=5 ; s=0.1 ; \tilde{M}_{j, \tau}=€ 100 ; m^{h}=$ $€ 0.1 /$ household.
2. Transport network parameters: $c_{1}^{0}=3,000 \mathrm{vph}$, $c_{2}^{0}=0 \mathrm{vph} ; c_{4}^{0}=3,000 \mathrm{vph} ; u_{1}=u_{2}=u_{4}=6,000$ vph; $t_{1}^{0}=t_{2}^{0}=5$ hours; $t_{4}^{0}=7$ hours $t_{3}^{0}=4$ hours; $s=1,500 \mathrm{vph}$.

3. Transit's operation and maintenance cost in period $\tau: Y_{\tau}^{2}=€ 1,000,000$.

4. Parameters of improvement cost functions: $\bar{b}_{2,1}=$ $1, b_{2,0}=€ 2,000$.

5. Parameters of maintenance cost functions: $\beta_{2,0}=$ $€ 1,200, \beta_{2,1}=€ 0.001, \beta_{2,2}=1$.

6. Parameters in travel cost functions: Value of time, $\psi=€ 15 / \mathrm{h}$; mode-specific cost, $\theta^{\text {car }}=16 ; \theta^{\text {transit }}=$ $30 ; \bar{\beta}=0.05 €^{-1}$.

7. Interest and inflation rates: $\tilde{i}=0.03 ; \tilde{j}=0.01$.

8. Converting factor: $n=365$ days $\times 24$ hours $\times 10$ years $=87,600$ hours/period.

9. Length of each period: 10 years.

10. Planning horizon and franchised period: $[0,50]$.

11. Specific parameters for each scheme:

a. BOT: The transit fare on link 3 between OD pair E1-R2 in period $\tau, p_{3,12, \tau}^{2}=€ 40$; the toll on link $1, \rho_{1, \tau}=€ 0$; Maximum allowable toll: $\rho_{\max }=€ 5$.

b. Cost recovery: The transit fare on link 3 , $p_{3,12, \tau}^{2}=€ 40$; the toll on link $1, \rho_{1, \tau}=€ 0$.

c. Cross-subsidization: The tolls on both links 1 and $2, \rho_{1, \tau}=\rho_{2, \tau}=€ 0$.

These values are chosen for illustrative purposes.

\subsection{Performance of each scheme}

The optimal designs under the three schemes are obtained by solving the PM model, the CR model, and the CS model using PREMIUM SOLVER PLATFORM. The corresponding performance measures are shown in Table 1 and Figure 2. In general, they show that road constructions have different impacts on related parties, including road users, private landowners, transit operators, private toll road operators, and the government. 
Table 1

The objective measure of each party under three schemes

\begin{tabular}{|c|c|c|c|c|c|}
\hline \multirow[b]{2}{*}{ Party } & \multirow[b]{2}{*}{ Objective measure } & \multirow{2}{*}{$\begin{array}{l}\text { Build-operate- } \\
\text { transfer (BOT) } \\
\text { (without equity) }\end{array}$} & \multicolumn{2}{|c|}{ Exact cost recovery } & \multirow{2}{*}{$\begin{array}{c}\text { Cross- } \\
\text { subsidization } \\
\text { (without equity) }\end{array}$} \\
\hline & & & Without equity & With equity & \\
\hline \multirow[t]{2}{*}{ Landowners } & $\begin{array}{l}\text { Change in profit of landowner } 2 \\
\text { (in million } € \text { ) }\end{array}$ & -937 & $-1,030$ & 0 & -112 \\
\hline & $\begin{array}{l}\text { Change in profit of landowner } 3 \\
\text { (in million } € \text { ) }\end{array}$ & 937 & 1,030 & 0 & 112 \\
\hline \multirow[t]{3}{*}{ Toll road operator } & Profit of toll road operator & 781 & 0 & 0 & 0 \\
\hline & $\begin{array}{l}\text { Construction and maintenance cost } \\
\text { (in million } € \text { ) }\end{array}$ & 360 & 360 & 6,030 & 360 \\
\hline & Toll revenue (in million $€$ ) & 1,141 & 360 & 6,030 & 0 \\
\hline Transit operator & Change in profit (in million $€$ ) & 2,680 & 2,490 & 5,120 & 484 \\
\hline \multirow[t]{3}{*}{ Government } & $\Delta \mathrm{SS}$ (in million $€$ ) & 9,580 & 9,370 & 4,360 & 16,100 \\
\hline & $\triangle \mathrm{CS}$ (in million $€$ ) & 6,120 & 6,880 & -765 & 16,000 \\
\hline & Average network travel time (min) & 358 & 358 & 371 & 320 \\
\hline
\end{tabular}

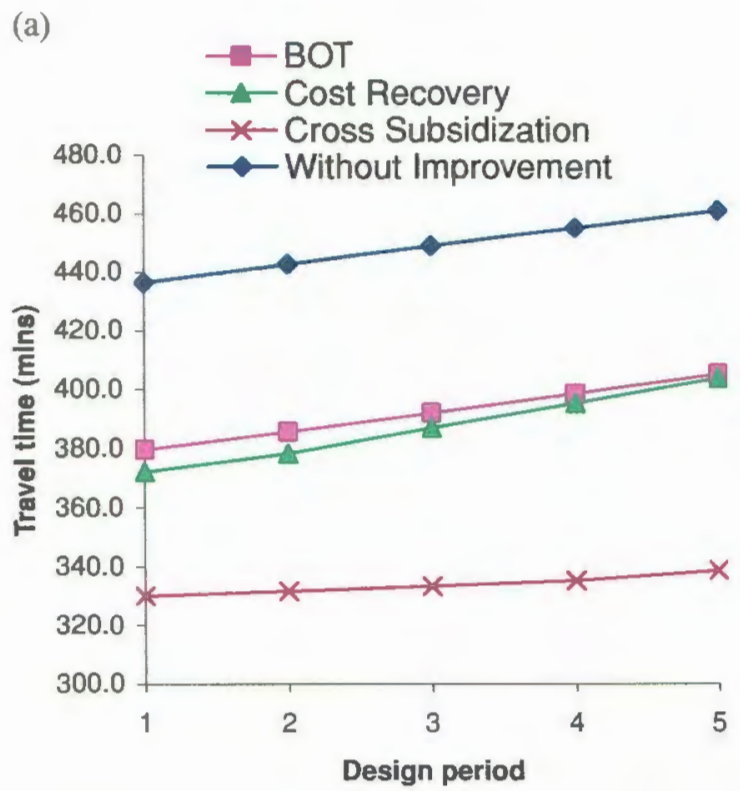

(b)

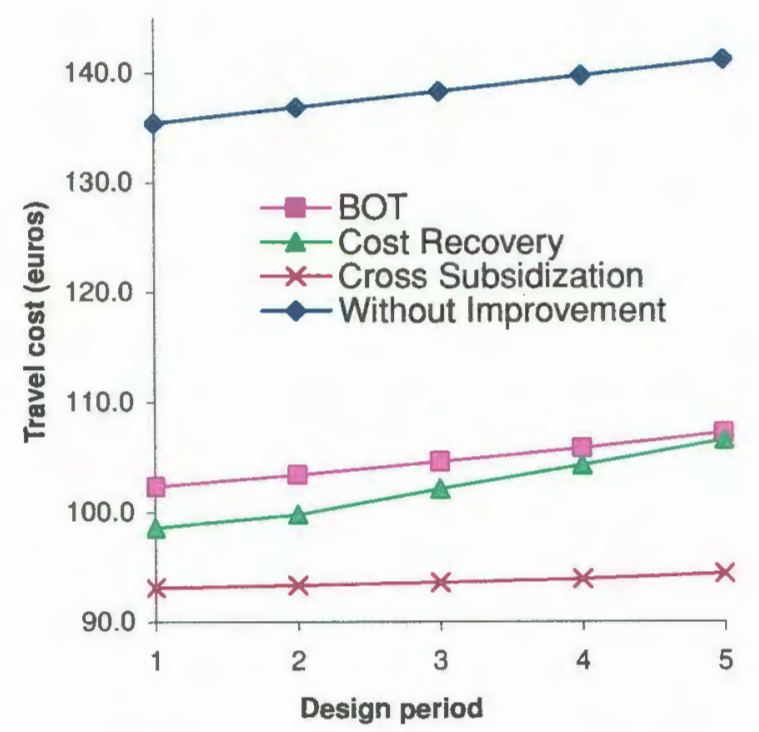

Fig. 2. Travel time and travel cost over time. (a) Travel time between OD pair E1-R2 and (b) travel cost between OD pair E1-R3.

3.2.1 Road users. They are concerned with their travel times and travel costs. According to Figure 2, the travel times and travel costs increase over time due to increase in population over time and increase in travel demand. However, after the implementation of any road construction projects, travel time and travel cost are less than those before.

3.2.2 Private landowners. Private landowners are concerned with their own profit. As shown in Table 1, without considering the equity of landowners, the profit of landowner 2 will be reduced but that of landowner 3 will be increased if any one of the schemes is implemented. Landowner 2 will object to any implementation unless the government provides landowner 2 a subsidy to raise the profit back to the original level.

3.2.3 Toll road operators. Private toll road operators are concerned with the profit from the project. Without considering the equity of landowners, the BOT scheme will result in generating a profit, but this profit may not be too attractive as the rate of return (i.e., toll revenue/construction and maintenance cost) is about $3 \%$, which is less than the usual norm of $10 \%-15 \%$. From 
the viewpoint of the private toll road operator, the project is not attractive if no subsidy is further given from the government. However, if the government gives the operator a subsidy to raise the rate of return to the minimum of $10 \%$, the SS will decrease further.

It is worthwhile to point out that in the cost recovery scheme, the builder and operator is the government, whose objective is to maximize the change in SS subject to cost recovery. As shown in Table 1, the profit is zero and the construction cost and maintenance cost is equal to the toll revenue.

3.2.4 Transit operators. When the transit operator is private and profit driven, without considering the equity of landowners the operator will welcome the implementation of the BOT and the cost recovery scheme because both schemes will lead to a higher transit profit. In particular, the operator will prefer the BOT scheme more as the change is larger.

When the transit operator is the government, the positive change in transit profit means that the implementation is good to society, as the change in transit profit (or the change in transit revenue minus the change in the operation and maintenance cost of transit) is part of the change in SS. Note that under cross-subsidization, the change in transit profit is greater than the construction and maintenance cost of toll roads.

3.2.5 Government. From the government's perspective, the three schemes are beneficial to society, as the change in social surplus ( $\Delta \mathrm{SS})$ is positive. In addition, from the viewpoint of congestion or road network performance, the three schemes do improve the situation, because the change in consumer surplus ( $\triangle \mathrm{CS})$ is positive and the average network travel time is lower than 466 minutes which is the average network travel time without the implementation of any scheme. However, the government needs to consider the unequal change in profit between the landowners when any one of these schemes is implemented and may require to subsidize the private toll road operator when the BOT scheme is implemented.

It is difficult to comment which scheme is the best in general after considering the perspectives of all the above parties. In this particular example, the crosssubsidization gives the highest $\Delta \mathrm{SS}$ and $\Delta \mathrm{CS}$ and the lowest average network travel time. This scheme is the best from the government point of view. The BOT scheme gives the largest profit from toll and transit revenue, and is the best from the viewpoint of the private transit and toll road operator. When the transit operator is private, cross-subsidization is not possible and BOT gives a better performance in terms of $\Delta S S$. Thus, BOT is the second best. However, all these observa- tions and conclusions are based on this specific case, and cannot be generalized to another study. Nevertheless, a general observation can be made. All schemes can lead to an unequal change in landowner profit, and some landowner's profit can be reduced. Landowners will object to the implementation of the scheme if this happens. To avoid this happening, we have to take their consideration into account when designing road construction (and improvement) projects.

\subsection{Performance of the cost recovery design under landowner equity consideration}

To deal with the consideration of landowners, we can add equity constraints to the three models, ensuring that the changes in landowner profits are nonnegative. For illustrative purposes, we only add equity constraints to the CR model to form the CR-equity model. This CR-equity model is solved by PREMIUM SOLVER PLATFORM, and the performance measures with and without the considerations of landowner equity are also provided in Table 1.

These results clearly show trade-offs between the perspectives of each of the parties. The equity scheme is worse than the original cost recovery design in terms of $\Delta S S, \Delta C S$, and the average network travel time. $\triangle S S$ and $\triangle C S$ are smaller and the average network travel time is higher, meaning that society receives less benefit and the road network is more congested when landowner equity is ensured. In particular, $\triangle \mathrm{CS}$ is negative, which is highly unacceptable. Road travelers face higher travel time and cost compared with the situation without considering equity. However, the private transit operator will favor the equity scheme as the change in profit is larger.

To illustrate the trade-offs further, we plot Figure 3, which is obtained by solving the CR model while setting tolls to be constant during the planning horizon. This figure demonstrates that the government has to increase the toll level from the optimal value of $€ 2$ so as to reduce the decrease in profit of landowner 2 , or to

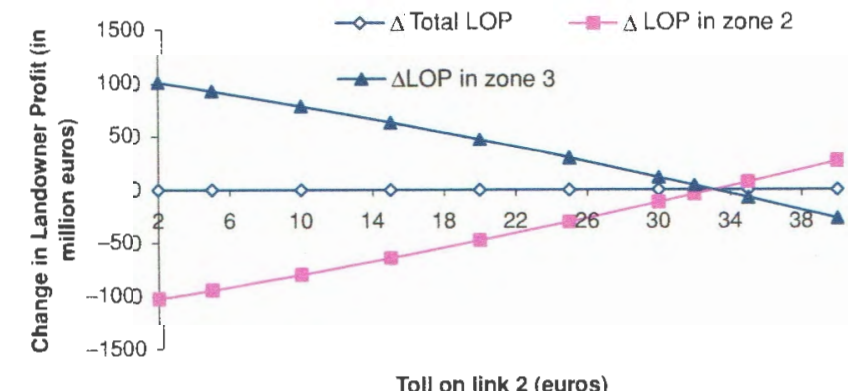

Fig. 3. Changes in landowner profits against toll on link 2. 
minimize the gap between the two changes in landowner profits. Moreover, in the extreme case, if the landowner equity is ensured, the toll charge is $€ 33$, which may be too high and may be objected by road travelers.

\section{CONCLUSIONS}

This article proposes a single-objective discrete network design framework considering the land-use transport interaction over time. This framework allows the evaluation of the impact of the design on related parties including landowners and contrasts to existing models that cannot be used for such purpose as the land usetransport interaction over time is not captured. This flexibility helps network planners and private firms with decision making. The proposed framework is formulated as a single-level maximization program, and can be solved by many existing optimization methods. Through the hybrid approach, the framework is also extended to consider multi-objectives. This multi-objective framework can aid the government making decisions considering the objective of each related party and eliminating a large number of alternative designs without trial and error.

This article also illustrates the models and the impacts of road constructions on related parties, especially landowners, under different network design schemes through a simple example, although the models can be applied to general networks. The results show that it is difficult to comment which scheme is the best in general after considering each party's perspective and that trade-offs exist between the objectives of all related parties. Moreover, all schemes lead to unequal changes in landowner profits. This raises the issue of landowner equity. If we aim at ensuring that their profits must not be reduced, other considerations such as societal benefit and the road network performance may get worse. Therefore, the government has to carefully consider the trade-offs.

This article opens up many research directions. First, this article does not consider heterogeneous values of time, mixed routing strategies, and the elasticities of housing demand and supply. One can incorporate them into the proposed frameworks in future studies. Second, the proposed frameworks do not consider demand and supply uncertainty, but in reality demand and supply are uncertain. Extending the proposed frameworks to capture uncertainties is definitely one important future research direction. Third, the Lowry land-use model incorporated into our proposed frameworks can serve the purposes of this article, but this model has some limitations such as ignoring the economic dimension and long-term character of location decisions. In the future, one can incorporate a more advanced land-use model into our proposed frameworks to study other important network-design-related issues, for instance, the effect of road network design on location decisions. Fourth, our proposed multi-objective framework can indeed incorporate sustainability objectives and indicators summarized by Litman (2008) into road network design analysis with the objective functions to be the desirable sustainability indicators and with the target value to be the desirable sustainability indicator values. One direction is therefore to apply this framework to analyze the trade-off between various indicators in road network design. Fifth, the proposed frameworks do not capture the effect of physical queuing on junction blockage, which can highly affect the accuracy of travel time estimation and bottleneck identification as pointed out in Lo and Szeto $(2004,2005)$. However, in the future, one can integrate a dynamic network loading model like the one in Nie et al. (2008) with the proposed frameworks by introducing another time dimension for within-day dynamics. Finally, the proposed models are path-based, which are not suitable for solving large networks involving many paths, because there are too many decision variables and it is time-consuming to find out all paths. Moreover, like other optimization problems, the proposed problem is highly nonconvex, which is difficult to solve for global solutions efficiently. Therefore, developing a link-based formulation and an efficient global optimization technique based on one or more heuristics like tabu search (e.g., Fan and Machemehl, 2008), genetic algorithms (e.g., $\mathrm{Ng}$ et al., 2009), and ant colony heuristics (e.g., Vitins and Axhausen, 2009) for this problem represents another worthy research direction.

\section{ACKNOWLEDGMENTS}

This research is jointly funded by the grant (201001159008) from the University Research Committee of the University of Hong Kong and the Programme for Research in Third-Level Institutions (PRTLI) administered by the Irish Higher Education Authority. The authors are grateful for the constructive comments of the referees.

\section{REFERENCES}

Abdulaal, M. \& LeBlanc, L. J. (1979), Continuous equilibrium network design models, Transportation Research Part B, 13, 19-32.

Ban, J. X., Liu, H. X., Ferris, M. C. \& Ran, B. (2006), A general MPCC model and its solution algorithm for continuous network design problem, Mathematical and Computer Modelling, 43, 493-505. 
Ben Akiva, M. \& Lerman, S. R. (1987), Discrete Choice Analysis, MIT Press, Cambridge, MA.

Boyce, D. E. (1984), Urban transportation network equilibrium and design models: Recent achievements and future prospects, Environment and Planning A, 16(11), 144574.

Boyce, D. E. \& Janson, B. N. (1980), A discrete transportation network design problem with combined trip distribution and assignment, Transportation Research Part B, 14, 147-54.

Bureau of Transport Economics (1998), Working Paper 39: Urban Transport Models: A Review. Department of Transport and Regional Services, Canberra, Australia.

Chen, A. \& Yang, C. (2004), Stochastic transportation network design problem with spatial equity constraint, Transportation Research Record, 1882, 97-104.

Chen, M. Y. \& Alfa, A. S. (1991), A network design algorithm using a stochastic incremental traffic assignment approach, Transportation Science, 25, 215-24.

Chiou, S. W. (2005), Bilevel programming for the continuous transport network design problem, Transportation $R e$ search Part $B, 39,361-83$.

Chiou, S. W. (2007), A generalized iterative scheme for network design problem, Applied Mathematics and Computation, 188(2), 1115-23.

Chiou, S. W. (2009), A subgradient optimization model for continuous road network design problem, Applied Mathematical Modelling, 33, 1386-96.

Davis, G. A. (1994), Exact local solution of the continuous network design problem via stochastic user equilibrium assignment. Transportation Research Part B, 28, 61-75.

Fan, W. \& Machemehl, R. B. (2008), Tabu search strategies for the public transportation network optimizations with variable transit demand, Computer-Aided Civil and Infrastructure Engineering, 23(7), 502-20.

Feng, C. M. \& Lin, J. J. (1999), A land use-network design model to generate alternative sketch maps for urban planning, Journal of the Eastern Asia Society for Transportation Studies, 3(4), 71-86.

Friesz, T. L. (1985), Transportation network equilibrium, design and aggregation: Key developments and research opportunities, Transportation Research Part A, 19(5-6), 41327.

Friesz, T. L., Anandalingam, G., Mehta, N. J., Nam, K., Shah, S. J. \& Tobin, R. L. (1993), The multiobjective equilibrium network design problem revisited: A simulated annealing approach, European Journal of Operational Research, 65, 44-57.

Friesz, T. L. \& Shah, S. (2001), An overview of nontraditional formulations of static and dynamic equilibrium network design, Transportation Research Part B, 35, 5-21.

Heydecker, B. G. (2002), Dynamic equilibrium network design, in M. A. P. Taylor (ed.). Transportation and Traffic Theory, Elsevier Science, Oxford, UK, pp. 349-70.

LeBlanc, L. J. (1975), An algorithm for discrete network design problem, Transportation Science, 9, 183-99.

Lee, D. H., Wu, L. \& Meng, Q. (2006), Equity based land-use and transportation problem, Journal of Advanced Trans. portation, 40(1), 75-93.

Lin, J. J. \& Feng, C. M. (2003), A bi-level programming model for the land use-network design problem, The Annals of Regional Science, 37, 93-105.

Litman, T. (2008), Well Measured: Developing Indicators for Comprehensive and Sustainable Transport Planning,
Victoria Transport Policy Institute (www.vtpi.org); at www.vtpi.org/wellmeas.pdf.

Lo, H. K. \& Szeto, W. Y. (2004), Modeling advanced traveler information services: Static versus dynamic paradigms, Transportation Research, 38B, 495-515.

Lo, H. K. \& Szeto, W. Y. (2005). Road pricing modeling for hyper-congestion, Transportation Research, 39A(7-9), 70522.

Lo, H. K. \& Szeto, W. Y. (2009), Time-dependent transport network design under cost-recovery, Transportation $R e$ search Part B, 43, 142-58.

Los, M. (1978), Simultaneous optimization of land use and transportation: A synthesis of the quadratic assignment problem and the optimal network problem, Regional Science and Urban Economics, 8, 21-42.

Los, M. (1979), A discrete-convex programming approach to the simultaneous optimization of land use and transportation, Transportation Research Part B, 13(1), 33-48.

Lowry, I. S. (1964), A Model of Metropolis, Report RM 4035 RC, Rand Corporation, Santa Monica, CA.

Magnanti, T. L. \& Wong, R. T. (1984), Network design and transportation planning: Model and algorithm, Transportation Science, 18(1), 1-55.

Marcotte, P. (1986), Network design problem with congestion effects: A case of bilevel programming, Mathematical Programming, 34, 142-62.

Meng, Q., Yang, H. \& Bell, M. G. H. (2001), An equivalent continuously differentiable model and a locally convergent algorithm for the continuous network design problem, Transportation Research Part B, 35, 83-105.

Meng, Q., Yang, H. \& Wong, S. C. (2000), A combined land-use and transportation model for work trips, Environment and Planning B: Planning and Design, 27(1), 93103.

Ng, M. W., Lin, D. Y. \& Waller, S. T. (2009), Optimal longterm infrastructure maintenance planning accounting for traffic dynamics, Computer-Aided Civil and Infrastructure Engineering, 24(7), 459-69.

Nie, Y., Ma, J. \& Zhang, H. M. (2008), A polymorphic dynamic network loading model, Computer-Aided Civil and Infrastructure Engineering, 23(2), 86-103.

Peiser, R. B. (1984), Land use versus road network design in community transport cost evaluation, Land Economics, 60 , 95-109.

Qiu, Y. \& Chen, S. (2007), Bi-level programming for continuous network design of comprehensive transportation system based on external optimization, in Proceedings of 2007 IEEE International Conference on Grey Systems and Intelligent Services, 1186-90.

Smith, J. M. \& Liebman, J. S. (1978), A zero-one integerprogramming formulation of the problem of land-use assignment and transportation-network design, Environment and Planning $B, 5(1), 101-15$.

Szeto, W. Y. \& Lo, H. K. (2006), Transportation network improvement and tolling strategies: The issue of intergeneration equity, Transportation Research Part A, 40, 227-43.

Szeto, W. Y. \& Lo, H. K. (2008), Time-dependent transport network improvement and tolling strategies, Transportation Research Part A, 42(2), 376-91.

Unnikrishnan, A., Valsaraj, V., Damnjanovic, I. \& Waller, S. T. (2009), Design and management strategies for mixed public private transportation networks: A meta-heuristic approach, Computer-Aided Civil and Infrastructure Engineering, 24(4), 266-79. 
Vitins, B. J. \& Axhausen, K. W. (2009), Optimization of large transport networks using the ant colony heuristic, Computer-Aided Civil and Infrastructure Engineering, 24(1), 1-14.

Wardrop, J. (1952), Some theoretical aspects of road traffic research, in Proceedings of the Institute of Civil Engineers, Part II, 325-78.

Williams, H. (1976), Travel demand models, duality relations and user bcncfit analysis, Journal of Regional Science, 16(2), $147-66$.
Williams, H. C. W. L. (1977), On the formation of travel demand models and economic evaluation measures of user benefit, Environment and Planning A, 9(3), 285344.

Wilson, A. G. (1970), Entropy in Urban and Regional Modelling, Pion, London.

Yang, H. \& Bell, M. G. H. (1998), Models and algorithms for road network design: A review and some new developments. Transport Reviews, 18(3), 25778 . 